This item was submitted to Loughborough's Research Repository by the author.

Items in Figshare are protected by copyright, with all rights reserved, unless otherwise indicated.

\title{
Point mobility of a cylindrical plate incorporating a tapered hole of power-law profile
}

PLEASE CITE THE PUBLISHED VERSION

http://dx.doi.org/10.1121/1.3585844

PUBLISHER

(C) Acoustical Society of America

VERSION

AM (Accepted Manuscript)

LICENCE

CC BY-NC-ND 4.0

REPOSITORY RECORD

O'Boy, D.J., E.P. Bowyer, and Victor V. Krylov. 2012. "Point Mobility of a Cylindrical Plate Incorporating a Tapered Hole of Power-law Profile". figshare. https://hdl.handle.net/2134/9956. 
This item was submitted to Loughborough's Institutional Repository (https://dspace.lboro.ac.uk/) by the author and is made available under the following Creative Commons Licence conditions.

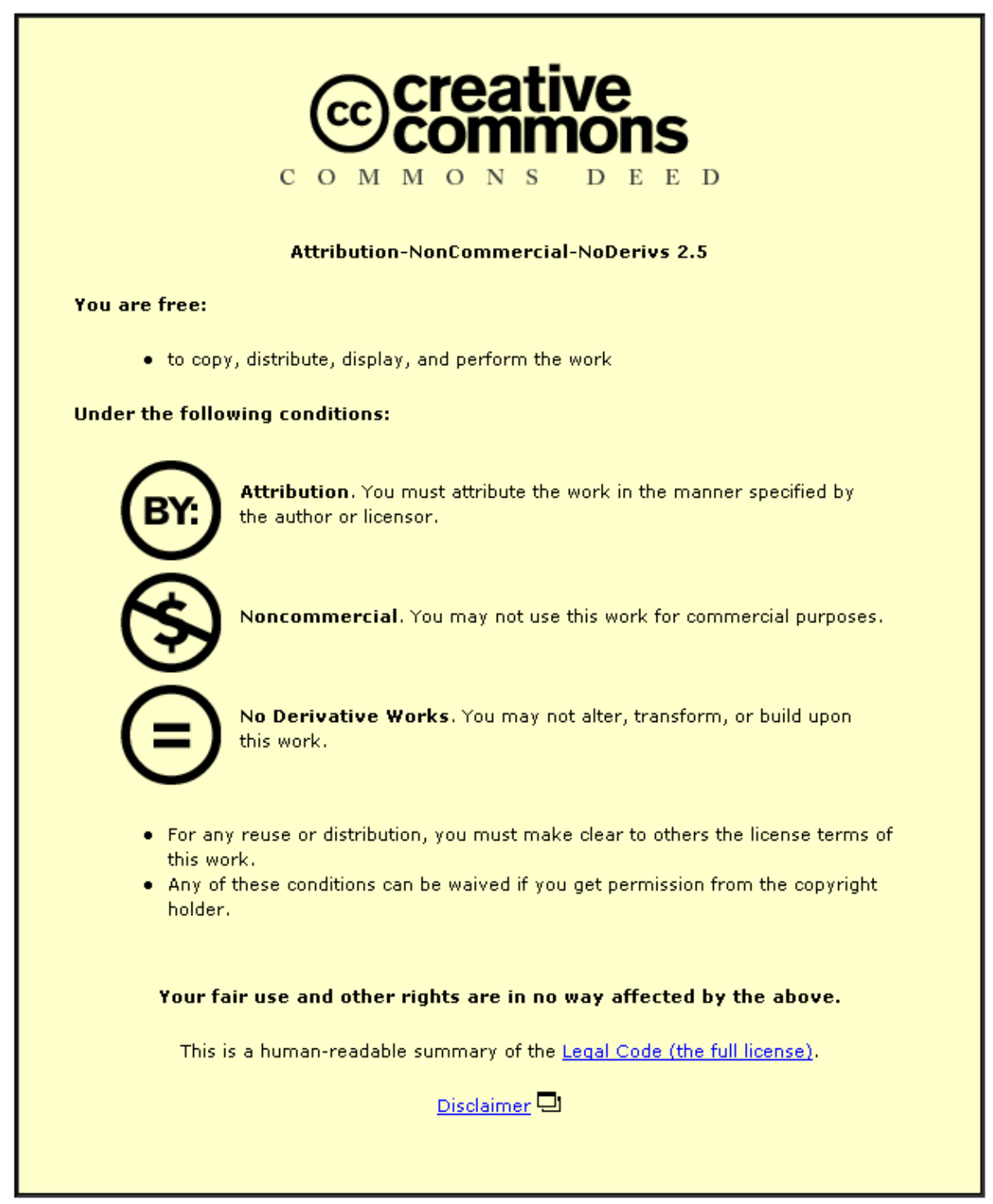

For the full text of this licence, please go to: http://creativecommons.org/licenses/by-nc-nd/2.5/ 


\title{
Point mobility of a cylindrical plate incorporating a tapered hole of power-law profile
}

\author{
Daniel J. O’Boy, ${ }^{\text {a) }}$ Elizabeth P. Bowyer, and Victor V. Krylov \\ Department of Aeronautical and Automotive Engineering, \\ Loughborough University, \\ Loughborough, \\ Leicestershire LE11 3TU, \\ United Kingdom.
}

(Dated: June 30, 2010)

Mobility measurements of a tapered plate 1 


\begin{abstract}
The paper describes the results of experimental measurements of point mobility carried out on circular plates containing tapered holes of quadratic power-law profile with attached damping layers. The obtained results are compared to the developed numerical model, as a means of validation. The profile of the tapered holes in the plates are designed to replicate the so called "acoustic black hole effect" associated with zero reflection of quasi-plane waves from a tapered hole in geometrical acoustics approximation. The driving point mobility measurements are provided, showing a comparison of the results for a constant thickness circular plate, a constant thickness plate with a layer of damping film applied and a plate with a quadratic power-law profile machined into the center, which is tested with a thin layer of elastic damping material attached. The results indicate a substantial suppression of resonant peaks. This agrees with a numerical model, which is based on the analytical solution available for the vibration of a plate with a central quadratic power-law profile. The paper contains results for the case of free boundary conditions on all edges of the plates, with emphasis placed on the accuracy of numerical predictions of resonant frequencies and the amplitudes of vibration.
\end{abstract}

PACS numbers: 43.20.Jr, 43.40.At, 43.40.Dx, 43.40.Tm, 43.50.Gf, 43.55.Wk 


\section{INTRODUCTION}

The need for a reduction of undesirable flexural vibration in plate structures has led to a significant amount of research into both the prediction of plate natural frequencies and also different methods which can be employed to manipulate them away from excitation frequencies. Furthermore, many different damping methods can be applied to plate structures to limit the amplitude of harmful vibration resonances, with varying levels of success. The amplitude of flexural vibration of plates is directly related to the amplitude of radiated noise, which can be uncomfortable or distracting, contributing to environmental noise pollution, requiring efforts from manufacturers to reduce it or manipulate the peak resonant frequencies away from sensitive regions (for example on an A-weighting curve) or due to legislative limits.

It is well known that applying a damping material directly to a vibrating plate structure does not lead to a significant reduction in the amplitude of vibration, as the plate bending stiffness and damping will dominate the response ${ }^{12}$. However, it has been previously shown that as the thickness of the plate material decreases, the overall composite damping increases $^{34}$. In addition, just increasing the thickness of a damping layer or the loss factor on a constant thickness plate does not necessarily increase the total loss factor of the plate ${ }^{5}$. Indeed, increases in mass can be unacceptable in many engineering applications, including aerospace and space structures.

One method of reducing the impact of flexural vibrations has been suggested by Krylov, where an indentation of power-law profile with added damping layer is incorporated into the plate structure $^{67}$, in order to reduce the reflections of flexural waves away from free edges of the structure. Even if a small layer of damping is applied to this profile, significant reductions in vibration amplitude can be achieved ${ }^{1678}$, as demonstrated on beams and rectangular plates. The incorporation of damped power-law profiles into elliptical plates have been studied by Gautier ${ }^{9}$ where reductions in mobility are seen on the plate when the forcing is

a)Electronic address: d.j.oboy@lboro.ac.uk 
in the focus of the profile.

The reduction in thickness of the plate should be optimized such that the flexural wave reflection from the change in cross section is small and yet large enough to obtain a significant composite loss factor when a damping film is applied to the surface. In considering flexural wave propagation through a plate of variable thickness changing in one dimension $x$ according to a power-law, $h(x)=\epsilon x^{m}$ (where $\epsilon$ and $m$ are positive constants), a geometrical acoustics approximation has been $\mathrm{used}^{67}$ to show that when no truncation is present in a profile of $m \geq 2$ then the incident wave never reaches the end of the profile and therefore becomes trapped near to the free edge $\left(\right.$ see also $\left.^{10}\right)$. However, as with all manufactured profiles, a truncation is introduced by machining tolerances and this truncation in the profile causes a large increase in the reflection coefficient, of the order 50-70 percent ${ }^{7}$. The addition of a damping layer reduces the amplitude of the reflected wave very efficiently due to the decreased thickness, leading to an overall reduction in the plate vibration amplitude. Such reduction of reflected waves has been termed the "acoustic black hole effect" 6789 as from a point outside of the profile a flexural wave may travel into the profile but ideally never return, having been attenuated by the efficient damping applied to the surface.

As the flexural waves travel into the profile, with a reduction in thickness, the amplitude of the flexural wave will increase and the phase speed decrease. Any damping layer attached to the surface is subject to larger displacement amplitude from the neutral layer with smaller wavelengths and as the total amount of energy converted into heat is proportional to the displacement, a reduction in plate thickness tends to be more efficient at attenuating energy.

Note that the assumptions of the geometrical acoustics approach lead to neglecting a number of terms in the equation of motion, which are relatively small. Although there are a significant number of analytical solutions to the bending plate equation of motion where the thickness is a constant, the additional complications of variable thickness mean that fewer complete analytical solutions are available, therefore the geometrical acoustics approach has been used on one dimensional profiles. The lack of analytical solutions has meant a reliance on numerical methods to obtain the natural frequencies of vibration of tapered beams and 
plates, see for example work by Gaines ${ }^{11}$ or Mabie ${ }^{1213}$, neither examining the integration of damping into the tapering thickness surface. However, in the case of the vibration of a cylindrical tapered plate, a fully analytical solution to the equation of motion is available from Conway $^{14}$, for the case of a quadratic variation in thickness $(m=2)$. This analytical solution does not neglect any terms and is therefore valid for all positions on the plate. It can be shown that the analytical solution for the quadratic variation in thickness shows the same asymptotic decrease in phase speed as an incident flexural wave traveling into a onedimensional wedge of quadratic power-law profile. As the analytical solution is available, it is possible to quantify the effect of including a damped cylindrical tapered indentation into a plate vibrating in flexure.

In the present paper, experimental measurements of the driving point mobility are presented in order to validate the theoretical results of a numerical model and to demonstrate the actual amplitude reductions and increased overall loss factor which can be achieved through the use of cylindrical power-law profiles in plate structures. Note that the bending plate equation of motion is only an approximate simple model which has frequency limitations and only ever provides the displacement due to one wave type. For higher accuracy it is necessary to utilize the full equations of elasticity, although this is then far more complex, especially where the thickness of the plate varies with location and as such, most work in this area is theoretical ${ }^{1516}$ with few experimental studies ${ }^{1718}$. The usual approach in this case is to consider the lowest modes of a Lamb wave, including symmetric and anti-symmetric modes in a plate whose thickness varies slowly with distance so that at any point, the Lamb wave analysis for constant thickness can be applied (these propagating waves in a slowly varying cross-sectional area are adiabatic waves where the wave continuously adapts to the change in local thickness through a modification of the wavenumber and phase velocity $)^{18}$. For certain flexural and longitudinal modes, there are points on the dispersion curves where it is possible to obtain zero group velocities for non-zero wavenumbers ${ }^{19}$ or backward wave transmission (where the phase velocity and group velocity are in opposite directions) ${ }^{20}$. The additional complexity illustrates the importance of simple models to predict the displace- 
ment amplitude at different frequencies for plate structures involving both constant and variable thickness geometries.

The cylindrical plate with a quadratic power-law profile in the center (referred to as a composite disc comprising a constant thickness annular disc and a smaller profiled disc) is shown in Fig. 1(a), with the notation used for the numerical model in Fig. 1(b) showing the radius of each truncation point and free edge. As the experimental measurements are compared directly to the findings from the numerical predictions, a short description of the numerical model is provided in section II. The analytical solution is examined and compared to the one dimensional case found in rectangular plates. The manufactured plates are discussed in section III, followed by a brief description of the experimental apparatus. The

results for the measured vibration are then presented and discussed in section IV, followed by conclusions (section V). The comparison will be made between a solid cylindrical disc, a solid cylindrical disc covered with a layer of viscoelastic damping material and a composite disc (comprising the undamped annular disc and a profiled indentation of quadratic power-law

shape, with a layer of viscoelastic damping film applied to the surface). The assessment of damping efficiency will take into account the overall peak amplitude mobility and individual loss factor at resonance (the latter measured through the half-power bandwidth method).

\section{NUMERICAL MODEL OF THE CYLINDRICAL BENDING PLATE WITH A QUADRATIC POWER-LAW INDENTATION}

In order to determine the vibration displacement of a composite plate made of an annulus of constant thickness with a profiled disc in the center, as shown in Fig. 1, we consider the two vibration problems separately and then join them with common boundary conditions using a wave based approach. The bending plate equation of motion for a constant thickness cylindrical plate is given by,

$$
-D \nabla^{4} w(r, \theta, t)=\rho h \frac{\partial^{2} w(r, \theta, t)}{\partial t^{2}}
$$




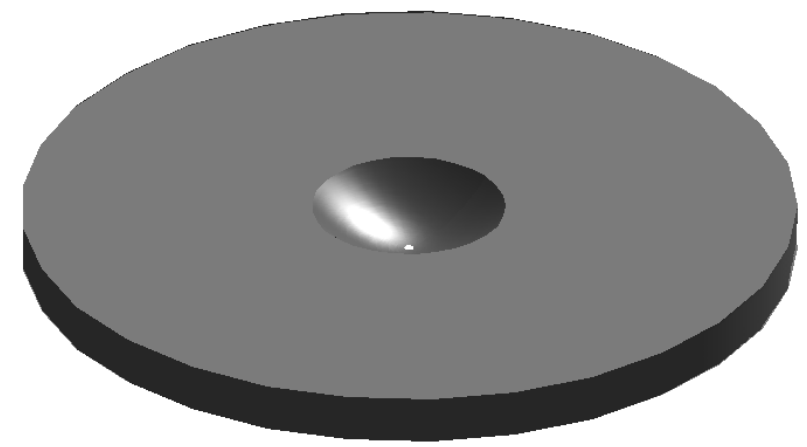

(a)

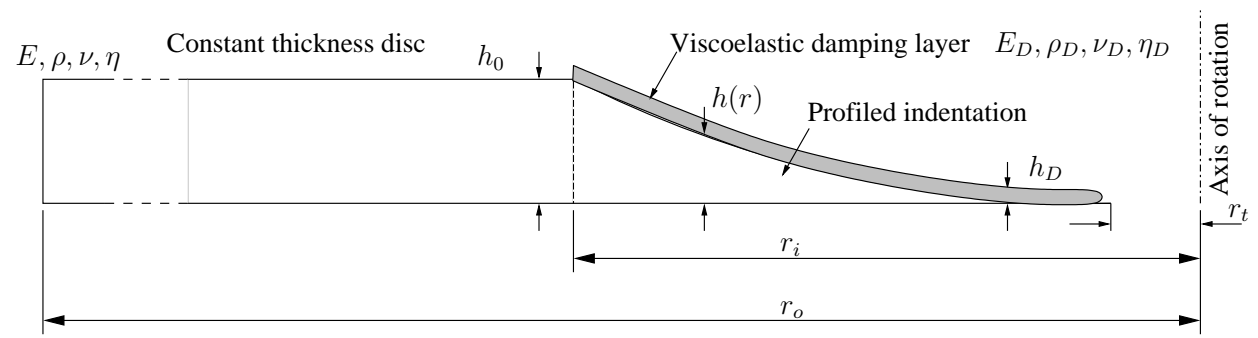

(b)

FIG. 1. Circular composite disc incorporating a quadratic power-law profile section in the center (not to scale), (a) illustration (b) notation for numerical model.

where $D=E h^{3} / 12\left(1-\nu^{2}\right)$ is the bending stiffness, $E, \rho$ and $\nu$ are the Young's modulus, density and Poisson's ratio and $h$ is the constant plate thickness. The plate displacement (at a normal to the surface) is $w(r, \theta, t)$, where we assume the Fourier transformations to angular order $n$ and frequency $\omega$ from the plate circumferential position and time respectively: $w(r, \theta, t)=w(r) e^{\mathrm{i} n \theta} e^{-\mathrm{i} \omega t}$. The differential operator $\nabla$ is applicable in polar coordinates where $r$ is the plate radius, $\nabla^{2} \nabla^{2}=\left(\frac{\partial^{2}}{\partial r^{2}}+\frac{1}{r} \frac{\partial}{\partial r}+\frac{1}{r^{2}} \frac{\partial^{2}}{\partial \theta^{2}}\right)\left(\frac{\partial^{2}}{\partial r^{2}}+\frac{1}{r} \frac{\partial}{\partial r}+\frac{1}{r^{2}} \frac{\partial^{2}}{\partial \theta^{2}}\right)$.

It may be shown that substitution of the form of the solution into the equation of motion leads to a solution for the displacement in terms of Bessel's functions ${ }^{2122}$.

$$
\begin{aligned}
w(r, \theta, t)= & \left(c_{1} \mathrm{~J}_{n}(\beta r)+c_{2} \mathrm{Y}_{n}(\beta r)+\right. \\
& \left.c_{3} \mathrm{~K}_{n}(\beta r)+c_{4} \mathrm{I}_{n}(\beta r)\right) e^{\mathrm{i} n \theta} e^{-\mathrm{i} \omega t},
\end{aligned}
$$


where $c_{1}, c_{2}, c_{3}, c_{4}$ are constants to be determined through the application of boundary conditions on the outer edge of the plate, at a radius $r=r_{o}$ and $\left.\beta^{2}=\sqrt{(} \rho h / D\right) \omega$. The Bessel's functions provide the solution to the displacement on any part of the constant thickness disc. If the constant thickness disc is solid, then two of these constants are zero, as the solutions are singular at $r=0$, however, should an inner radius be included at $r=r_{i}$, as is required for this model, all four constants are required and we have an inner free edge.

It now remains to find a solution for the bending plate equation of motion with a change in thickness with radial position only. This profiled indentation starts at a radius $r=r_{i}$ and continues to a truncation radius $r=r_{t}$ with the same material properties as the constant thickness disc described above. This center section has an equation of motion given by Harris $^{23}$, where the bending stiffness now varies with radial position,

$$
\begin{aligned}
\rho h(r) \frac{\partial^{2} w(r, \theta, t)}{\partial t^{2}}= & (1-v) \diamond^{4}\{D(r), w(r, \theta, t)\}- \\
& \nabla^{2}\left[D(r) \nabla^{2} w(r, \theta, t)\right],
\end{aligned}
$$

and $\diamond^{4}\{D(r), w(r, \theta, t)\}=\frac{\partial^{2} D}{\partial r^{2}}\left(\frac{1}{r} \frac{\partial w}{\partial r}+\frac{1}{r^{2}} \frac{\partial^{2} w}{\partial \theta^{2}}\right)+\frac{\partial^{2} w}{\partial r^{2}}\left(\frac{1}{r} \frac{\partial D}{\partial r}\right)$, is the bilinear operator in polar coordinates.

When the thickness profile is a quadratic power-law, $h(r)=\epsilon r^{2}$, an analytical solution is available from Conway ${ }^{14}$ by defining $K=12 \rho \omega^{2}\left(1-\nu^{2}\right) / E \epsilon^{2}$ which then yields the four solutions to the displacement as,

$$
w(r, n=0, \omega)=c_{5} r^{\lambda_{1}}+c_{6} r^{\lambda_{2}}+c_{7} r^{\lambda_{3}}+c_{8} r^{\lambda_{4}}
$$

where $\lambda_{1,2,3,4}=-2 \pm \sqrt{7-3 \nu \pm \sqrt{9\left(1-\nu^{2}\right)+K}}$, which is valid for the first angular order mode, $n=0$ although subsequent mode numbers can also be calculated numerically.

The predictions shown in this paper utilize estimates of the material parameters for the experimental steel plates, thus $E=210 \mathrm{GPa}, \rho=7800 \mathrm{~kg} / \mathrm{m}^{3}$ and $\nu=0.3$. The outer radius $r_{o}=0.25 \mathrm{~m}$, inner radius $r_{i}=0.1 \mathrm{~m}$, truncation radius $r_{t}=0.005 \mathrm{~m}$ and thickness $0.005 \mathrm{~m}$ with a frequency resolution of $4 \mathrm{~Hz}$. 


\section{A. Application of boundary conditions and solution method}

The form of Eqs. (2) and (4) both contain four constants requiring four boundary conditions to solve each discrete problem. These four conditions are plate displacement $w(r)$, slope $\partial w(r) / \partial r$, bending moment $M(r)$ and shear force $V(r)$, which can be arranged in matrix form $\mathbf{W}$ for application to a specific radius $r_{j}$ (with a matrix of associated constants $\mathbf{C}), \mathbf{W}_{r_{j}}=[w, \partial w / \partial r, M, V]_{r_{j}}^{\mathrm{T}}=\mathbf{A}_{r_{j}} \mathbf{C}$, where $\mathbf{A}$ is a four by four containing the material properties. The expressions for bending moment and shear force are zero at a free edge and are given by ${ }^{2122}$,

$$
\begin{aligned}
M(r)= & \frac{\partial^{2} w}{\partial r^{2}}+\nu\left(\frac{1}{r} \frac{\partial w}{\partial r}-\frac{n^{2}}{r^{2}} w\right), \\
V(r)= & \frac{\partial^{3} w}{\partial r^{3}}+\frac{1}{r} \frac{\partial^{2} w}{\partial r^{2}}- \\
& \frac{1}{r^{2}}\left(1+(2-\nu) n^{2}\right) \frac{\partial w}{\partial r}+\frac{(3-\nu) n^{2}}{r^{3}} w .
\end{aligned}
$$

If a layer $j$ can be defined by an outer radius $r_{j+1}$ and an inner radius $r_{j}$, then the outer boundary conditions can be related to the inner ones by matrix substitution,

$$
\mathbf{W}_{r_{j+1}}=\mathbf{A}_{r_{j+1}} \mathbf{A}_{r_{j}}^{-1} \mathbf{W}_{r_{j}}=\mathbf{X}_{j} \mathbf{W}_{r_{j}} .
$$

To connect a series of layers together, or join the constant thickness disc to an inner profile, common boundary conditions are assumed. At a specific radius we may also apply a harmonic excitation force, $p(r)$. Providing any four boundary conditions, Eq. (7) can be solved using a matrix shooting method to obtain the remaining unknown four conditions ${ }^{24}$ (neglecting the null solution). For an isotropic constant thickness disc (whose solution was listed in Eq. (2)), the functions of the outer radius can be directly related to the inner radius $\mathbf{W}_{r_{o}}=\mathbf{X}_{\text {const }} \mathbf{W}_{r_{i}}$. Similarly, for the isotropic wedge indentation $\mathbf{W}_{r_{i}}=\mathbf{X}_{\text {indent }} \mathbf{W}_{r_{t}}$. Coupling these together yields an overall matrix connecting the properties on the very outside of the composite plate to those on the inside.

$$
\mathbf{W}_{r_{o}}=\mathbf{X}_{\text {const }} \mathbf{X}_{\text {indent }} \mathbf{W}_{r_{t}} \text {. }
$$


The usual function of interest is the driving point mobility measurement, the frequency transfer function where the ratio of normal surface velocity $v=\dot{w}$ is co-located with the excitation force $p$ applied as an impulse, $\dot{w}(r) / p(r)$ (the dot represents a differentiation with respect to time). For a constant thickness annular disc, free edge boundary conditions are applied to the outer and inner radii, $M\left(r_{o}\right)=0, V\left(r_{o}\right)=0, M\left(r_{i}\right)=0, V\left(r_{i}\right)=0$. Where the disc contains a profiled indentation, common boundary conditions are applied at the join $r=r_{i}$ and the inner free edge condition is then applied to the truncation position $M\left(r_{o}\right)=0, V\left(r_{o}\right)=0, M\left(r_{t}\right)=0$ and $V\left(r_{t}\right)=0$.

\section{B. Loss factor of a cylindrical indentation of tapered profile}

In order to calculate appropriate driving point mobility results, the material must include some inherent damping (this is small for metal). In this paper, three plates are compared with different damping methodologies; (i) a solid circular plate with outer radius $r_{o}$ and only inherent damping, (ii) the same circular plate with a thin layer of viscoelastic damping film applied to the surface and (iii) the composite plate with a similar layer of damping film applied only to the surface of the wedge indentation.

The damping loss factor can be introduced through a complex Young's modulus ${ }^{252627}$ $E=E(1+\mathrm{i} \eta)$. The loss factor $\eta$ is a function of many variables, including frequency and temperature, however, if the applied stress is assumed to be small then Cremer et al. allow the loss factor for bending waves to be independent of frequency in the range of interest for this paper and structural plate dimensions ${ }^{25}$.

The inherent loss factor for a steel material at a temperature of approximately $18^{\circ} \mathrm{C}$ $\left(64^{\circ} \mathrm{F}\right)$ is estimated based on values provided in the literature as $\eta=0.004$, which is within the range given by DeSilva (0.002 to 0.01$)^{26}$, Mead (0.0005 to 0.01$)^{28}$ or Ross, Kerwin and Ungar (0.001 to 0.008$)^{4}$.

When a damping layer is applied to a stiff base plate, the composite loss factor $\eta_{\text {comp }}$ can be determined using the standard equations from Oberst $^{4}$, or their simplified form ${ }^{25}$, derived 
considering the equation of motion of a beam with applied viscoelastic layer with material subscripts $D$. Higher order forms of these Ross, Kerwin and Ungar equations are available ${ }^{428}$, however, given the approximate form of the loss factor used in this paper, these are considered not necessary. Similarly, more complex derivations are also available ${ }^{29}$, however, these also note that the simplified forms do agree well with experimental measurements.

$$
\begin{aligned}
\eta_{\text {comp }} & =\eta_{D} h_{D}\left(\left(h+h_{D}\right) / 2\right)^{2} / D^{\prime} \\
D^{\prime} & =E h^{3} / 12+E_{D} h_{D}\left(\left(h+h_{D}\right) / 2\right)^{2} .
\end{aligned}
$$

The damping layer applied to the surface of the constant thickness plate or wedge indentation in the experimental measurements is a commercial constrained layer foam of thickness $h_{D}=1.2 \mathrm{~mm}$, Young's modulus of approximately $E_{D}=100 \mathrm{MPa}$ and loss factor $\eta_{D}=2.0$ and will be treated as a single layer, in contrast to the constrained layer analysis by Kerwin ${ }^{27}$. This damping material has a high loss factor which compares well with similar polymer materials in the literature ${ }^{25}$. For example, Mead considers a typical polymer material which varies with excitation frequency, where the peak loss factor occurs between a frequency of 100-5000 Hz, decreasing rapidly above this value, coinciding with an increase in the Young's modulus as the polymer enters the glassy properties zone ${ }^{28}$.

The implementation of Eq. (9) with the polymer damping layer to the constant thickness disc yields a composite damping layer of $\eta_{\text {comp }}=0.00593$, an increase of 48 percent over the inherent damping of the metal plate.

For the wedge indentation from a radius $r_{i}$ to a truncation $r_{t}$, a single matrix can be used to connect the properties on either side, as in Eq. (8), however, if a viscoelastic damping layer is applied to the wedge surface, the local composite loss factor will change for every radial position. As the damping layer has a density far lower than the wedge material (for example mild steel), it is assumed that the mass is negligible and that the Young's modulus does not change the characteristic power-law profile of the wedge indentation.

The boundary conditions can then be written as a series of $J_{\max }$ thin slices, each of a 
slightly different composite loss factor.

$$
\mathbf{W}_{r_{i}}=\prod_{j=1}^{j=J_{\max }} \mathbf{X}_{j}\left(r_{j}, \eta\left(r_{j}\right)\right) \mathbf{W}_{r_{t}}
$$

The form of Eq. (10) takes into account both the analytical solution of a quadratic power-law profile indentation and also allows the different forms of viscoelastic damping, which are only applicable for constant thickness beams, to be applied to a wedge indentation of tapering thickness.

As most damping applications tend to be characterized in terms of a single loss factor, this can be determined for an equivalent wedge with loss factor $\eta_{\text {equiv }}$ which has the same energy absorption as a wedge with a thin layer of damping material applied but critically, does not vary with the radial position.

$$
\mathbf{X}_{j}\left(r_{j}, \eta_{\text {equiv }}\right)=\prod_{j=1}^{j=J_{\max }} \mathbf{X}_{j}\left(r_{j}, \eta\left(r_{j}\right)\right)
$$

The application of Eq. (11) for a wedge indentation covered by a layer of the polymer damping film causes a significant increase in the local composite loss factor, especially close to the profile truncation point. The equivalent loss factor for the whole damped wedge indentation is $\eta_{\text {equiv }}=0.148$, an increase of 3600 percent over the inherent damping available in the steel material. However, although this equivalent loss factor is high, it only applies to the area of the wedge indentation in the numerical prediction (which is small compared to the area of the plate), therefore the total loss factor for the composite plate will be significantly lower.

\section{Variation of flexural wave amplitude with position}

The form of the analytical solution for a quadratic profile, Eq. (4) demonstrates a singularity as the radius tends to zero, as the displacement has a form $\ln (r)$ which is not well defined at $r=0$. In addition, for positions close to $r=0$, the slope tends to the vertical in Eq. (8). This is the cylindrical equivalent of the "acoustic black hole effect" described in beams. 
In order to prevent this singularity, a truncation must be introduced at a radius $r=r_{t}$ in order to provide a complete free edge (a hole in the very center of the plate). Wang has stated that the amplitude increase grows so large without this hole, that the vibration could not occur and the resonant frequencies must therefore decrease to zero ${ }^{30}$. However, in this case it is likely that further terms for large scale elastic deformation would become important.

This increase in amplitude is illustrated in Fig. 2(a), which shows the amplitude of displacement $w(\omega, n=0, r) / p$ in $\mathrm{dB}$ as a dispersion curve for various radial positions on the plate with the $n=0$ vibration mode when no damping is applied to the plate surface. There appear increasing numbers of nodal points for higher frequencies when considering positions above $r=r_{i}$, however, on the surface of the wedge indentation, the amplitude grows rapidly and is $10 \mathrm{~dB}$ above the amplitude for the constant thickness section. The flexural wave, excited at a radius $r=r_{o}$ propagates through the constant thickness section, through the wedge indentation and reflects from the free edge at the truncation position. The sum of these incident and reflected propagating waves create the nodal pattern seen in Fig. 2(b) where the amplitude at a single resonant frequency has been illustrated. Any additional damping placed in the wedge indentation is subject to far higher amplitudes of motion with shorter wavelengths, thus increasing the efficiency of the attenuation. As the wave travels through the profiled indentation, the amplitude increases significantly and the phase speed decreases which reverses once the wave reflects from the free edge. Without the truncation, according to the simplified theory, the phase speed would continue to asymptotically decrease, however, through the introduction of a truncation radius, it is assumed in this paper that the amplitude of motion away from the truncation position is reflective of the real motion found in actual plate vibration. 


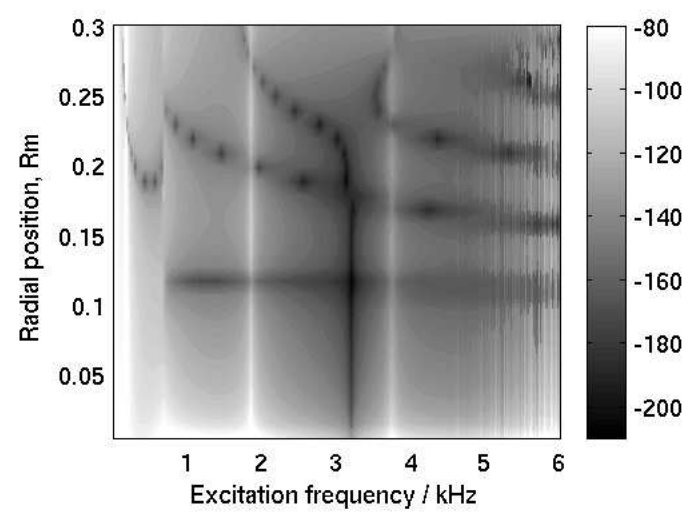

(a)

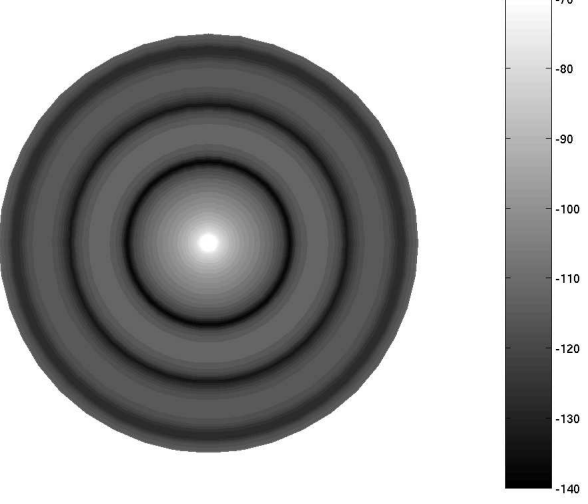

(b)

FIG. 2. Vibration amplitude $w(\omega, n=0, r) / p$ of a composite plate comprising an annulus with an undamped profiled indentation. The frequency dispersion curve is shown in (a) for different radial positions on the composite plate when excited in the $n=0$ mode. In (b) the displacement amplitude is shown for a single frequency of $1850 \mathrm{~Hz}$ and $n=0$, showing the nodal pattern created by the flexural waves traveling into the center of the plate, through the power-law profile and reflecting out from the free edge at the truncation point (the lighter the color, the higher the amplitude).

\section{EXPERIMENTAL MEASUREMENTS}

As a validation exercise, three cylindrical plates were manufactured out of mild steel with a nominal thickness of $5 \mathrm{~mm}$ via milling and an outer diameter of $500 \mathrm{~mm}$. The first is a constant thickness disc typical of some structural plates, the second is the constant thickness disc with a layer of damping film on the surface, the final one is the composite disc (the same outer diameter but with a profiled indentation of quadratic power-law from a diameter of $200 \mathrm{~mm}$ to a truncation diameter of $10 \mathrm{~mm}$, below this, the material is likely to tear). The profiled indentation has a thin layer of damping film applied to the surface.

The acceleration normal to the plate surface $a \mathrm{~m} / \mathrm{s}^{2}$, was measured using a standard Bruel and Kjaer accelerometer (series 4371) and post-processed into the normal velocity $v \mathrm{~m} / \mathrm{s}$ assuming harmonic excitation by the excitation frequency $\omega, v=a /(\mathrm{i} \omega)$. The plate 
was excited using a broadband electromagnetic shaker, with the force $p \mathrm{~N}$ measured through a Bruel and Kjaer force transducer (series 8200), positioned at a diameter of $300 \mathrm{~mm}$ from the center of the plate and attached firmly through adhesive.

The post-processed results were Fourier averaged (including the use of a Hanning filter) to obtain driving point mobility measurements (where the accelerometer and force transducer are co-located) with a frequency interval of $4 \mathrm{~Hz}$, between 0 and $6.05 \mathrm{kHz}$. All plates were suspended in a cradle via two lightweight wires to simulate free boundary conditions on the edges of the plate (the wires passed outside of the profiled section) at all times. The driving point mobilities $v / p \mathrm{~m} / \mathrm{s} / \mathrm{N}$ are shown on a decibel scale with a reference value of $1 \mathrm{~m} / \mathrm{s} / \mathrm{N}$.

The results for the reference constant thickness plate are shown in Fig. 3, comparing numerical predictions against experimental measurements. There is a good agreement between the predicted locations of the resonant frequencies and the experimental measurements, with the error less than five percent for most modes. The error increases slightly for higher frequencies, most likely due to the neglecting of shear deformation and rotary inertia terms in the equation of motion.

When a thin damping layer is introduced to the surface of the constant thickness plate, the amplitudes are reduced by an average of 1-4 $\mathrm{dB}$ at peak resonant frequencies, as the inherent damping in the plate dominates the response (as the plate extensional stiffness is so much higher than the damping layer).

When the damped indentation is incorporated into the plate, the numerical model indicates that the peak mobility amplitude will decrease due to a larger overall loss factor. This driving point mobility for the composite disc with a damped wedge indentation is shown in Fig. 4 comparing the experimental measurements with the numerical predictions. The peak mobility at higher frequencies is significantly decreased, potentially at the expense of an increase in the peak mobility at the lowest resonances.

For all three cases (two are shown), the numerical predictions are accurate (for frequency location) to within five percent which provides confidence in the model to predict other vibration results and damping configurations. The amplitude is more variable however and 
there is evidence that the numerical predictions can overestimate the damping at the highest frequencies.

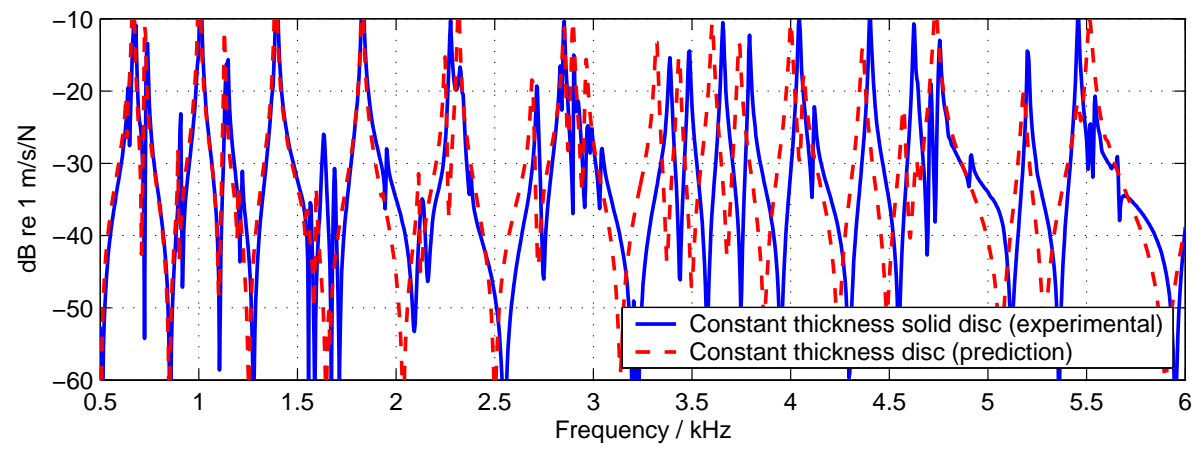

FIG. 3. Driving point mobility measurements $(v / p)$ dB showing the cylindrical plate of constant thickness for numerical predictions against experimental measurements (color online).

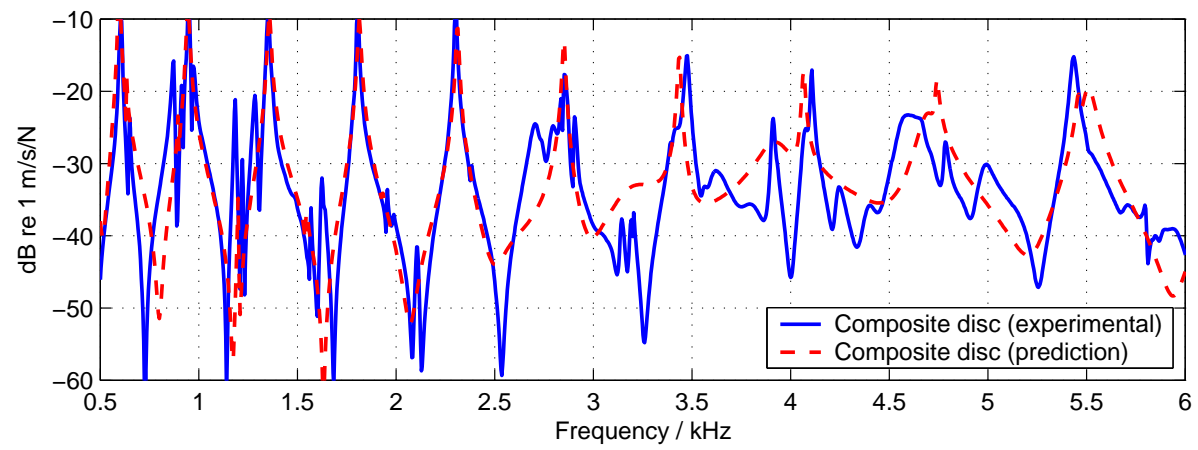

FIG. 4. Driving point mobility measurements $(v / p)$ dB showing the annular plate of constant thickness with a central damped wedge indentation of power-law profile, numerical predictions and experimental measurements (color online).

It remains to assess the accuracy of the total loss factor, carried out through an analysis of the half-power bandwidth of various resonant peaks, $\eta_{\text {Total }}=\Delta \omega_{3 d B} / \omega$, applicable for small values of the composite damping ${ }^{31}$ where the resonant modes can clearly be separated, providing the damping to be measured is greater than that introduced by boundary supports and attachment methods ${ }^{25}$. This analysis is derived from a single degree of freedom system, 
where only one resonance is present. At very high frequencies, the resonant peaks merge such that the individual bandwidth shapes cannot be clearly identified. This method provides an estimate of the total loss factor (which can be difficult to measure directly) and represents both the propagation losses and attenuation from free edge reflections, where the energy is converted from flexural to edge waves propagating along the edge of the plate (in the numerical predictions, we assume perfect reflection from the free edge). It also incorporates all losses from attachment of sensors, sound radiation and interaction with external supports at the boundary. The method has been used to successfully determine the loss factor for variously shaped plates, including corrugated surfaces which increase the total loss factor ${ }^{31}$, although the cited study only examined the first three modes of vibration.

The total loss factor $\eta_{\text {Total }}$ for the different plates are shown in Fig. 5(a), applied to the experimental measurements, both with data points and the best fit curve. At lower frequencies, the loss factor rises, as the material damping is proportional to the displacement (which is greater for lower modes of vibration) and loading ${ }^{31}$. For example, deSilva includes experimental measurements for general structural vibration indicating that a tenfold increase in the amplitude yields a 33 percent increase in the loss factor ${ }^{26}$. Although the loss factor shown in the best fit lines continues to increase as the frequency tends to zero, this is only due to the lowest point being equal to the lowest resonance. In reality, below the first resonance the loss factor increases as the square of frequency ${ }^{5}$.

With the above caveat, the half-power bandwidth method can be applied to the constant thickness disc and the damped constant thickness disc to determine the inherent loss factor and the damping material loss factor respectively. However, when applied to the frequency response curve for the composite disc, it is not possible to separate out the losses from the constant thickness section, the tapering section and the edge reflection (where the reflection coefficient is likely less than 100 percent). In addition, the method is limited by the small number of data points available from the resonances.

There is broadly good agreement in the relative magnitudes of the total loss factors, the trends are replicated with similar differences between the three plate configurations. 
The numerical predictions appear to taper to an asymptotic faster than the experimental measurements would indicate, possibly as the loss factor of the plate and damping film are initial approximations. It also appears that the estimated magnitude of the inherent damping estimated for the plate steel is larger than experimentally determined. Ungar notes that for very high frequencies, the total loss factor becomes independent of the damping material loss factor ${ }^{5}$ and we note that all graphs are asymptotically converging at high frequencies (above those considered in this study).

The largest overall damping is gained with the composite plate in both numerical predictions and experimental measurements, indicating the greater damping efficiency of the damped wedge profile.

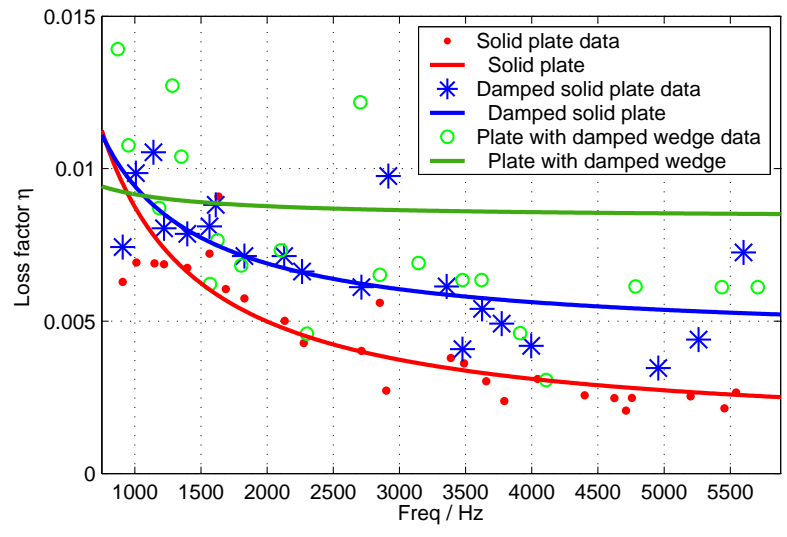

(a)

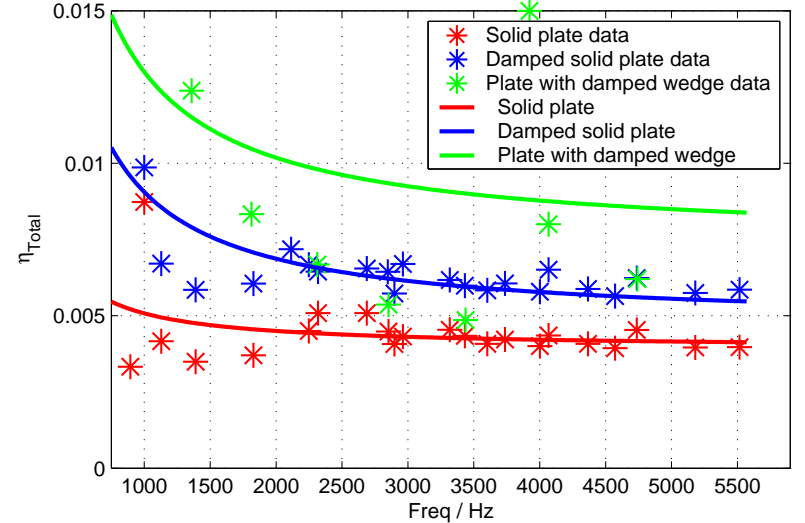

(b)

FIG. 5. Estimates of the total loss factor $\eta_{\text {Total }}$ for three different circular plates by the half-power bandwidth method (a) Experimental measurements, (b) Numerical predictions (color online).

\section{RESULTS AND DISCUSSION}

The inclusion of a tapered, damped profile has been shown to reduce the driving point mobility at a single point. Predictions are now presented for the mean average driving point mobility $\bar{v}$, for ten positions on the constant thickness section between $r=r_{o}$ and $r=r_{i}$, 
$\bar{v}=\frac{1}{10} \sum_{k=1}^{k=10} v\left(\omega, \theta=0, r_{k}\right) / p\left(r_{k}\right)$. These are shown for the three plate configurations in Fig. 6, where it may be seen that the highest average peak mobility occurs with the solid undamped plate, followed by the damped solid plate and finally, with the lowest overall peak mobility, the composite plate.

These averages show that the damped wedge indentation yields an average reduction of $4 \mathrm{~dB}$ over the solid damped plate. For example, at a frequency of $1.38 \mathrm{kHz}$, the peak amplitudes are $10.65 \mathrm{~dB}, 7.2 \mathrm{~dB}$ and $1.25 \mathrm{~dB}$ for the solid, damped solid and composite plates respectively. At $2.85 \mathrm{kHz}$, the values are 3.85, 0.9 and $0.06 \mathrm{~dB}$, while at higher frequencies, the potential amplitude reductions increase. For example, at $4.74 \mathrm{kHz}$, the peak mobility amplitudes are 4.5, 2 and $-2 \mathrm{~dB}$ while at $5.46 \mathrm{~dB}$ the amplitudes are 2.5, -0.5 and -3.5 respectively.

The maximum average reduction appears at a frequency of $3.32 \mathrm{kHz}$, where the solid plate has an amplitude of $4.6 \mathrm{~dB}$, with a reduction of $2.8 \mathrm{~dB}$ when a layer of damping film is applied. However, when the profiled indentation is incorporated into the plate, the reduction increases to 10.9 dB. Furthermore, several of the resonances appear to be smoothed out, see for example the regions at 1.65, 2.7 and $3.5-4 \mathrm{kHz}$.

Further work should examine the far-field radiated noise resulting from the plate vibration, in particular whether the reduction in noise from the vibration on the constant thickness section is less or greater than the noise resulting from the increase in vibration on the damped wedge indentation.

\section{CONCLUSIONS}

The experimental findings have illustrated that the incorporation of damped circular profiles of power-law design into plate structures can lead to a reduction in the amplitude of flexural vibration, especially at higher frequencies above the fundamental mode shapes.

With the attached damping layer, reductions of flexural vibration of $1-4 \mathrm{~dB}$ are possible for most frequencies while reductions of up to $10.9 \mathrm{~dB}$ are possible by incorporating a damped 


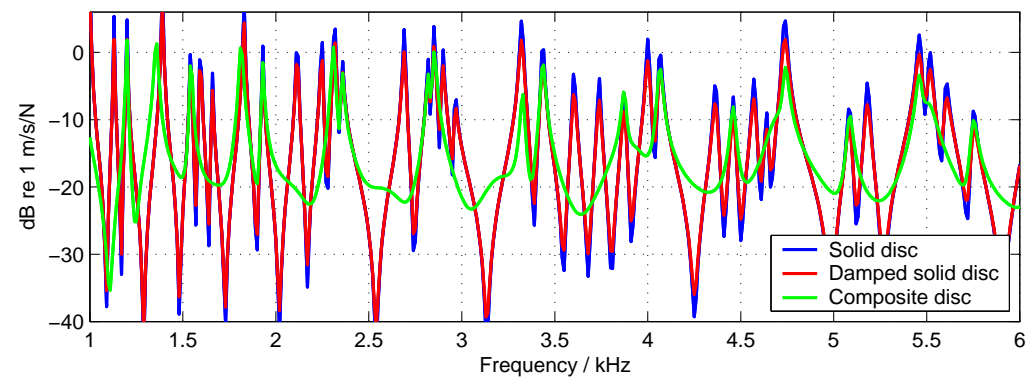

FIG. 6. Predictions of the average peak point mobility measurements $\bar{v}=\frac{1}{10} \sum_{k=1}^{k=10} v(\omega, \theta=$ $\left.0, r_{k}\right) / p\left(r_{k}\right) \mathrm{dB}$ (color online).

indentation of quadratic power-law into the plate.

The numerical model has been shown to provide accurate predictions of the composite plate natural frequencies above $1 \mathrm{kHz}$, when compared to experimental measurements. The natural frequencies above $1.5 \mathrm{kHz}$ vary by less than five percent providing confidence in the numerical model to design the plate profile to avoid specific excitation frequencies.

It has been shown through half power bandwidth measurements that the total loss factor for the composite plate is greater than the total loss factor when the solid plate is just covered by a layer of damping film. As the circular plate can seldom be used as a structural element as it is, further work should concentrate on incorporating damped tapered profiles into complex structures suffering from severe vibration and noise problems.

\section{Acknowledgments}

The research reported here has been supported by EPSRC grant EP/F009232/1.

\section{References}

1 D. J. O'Boy, V. V. Krylov, and V. Kralovic, "Damping of flexural vibrations in rectangular plates using the acoustic black hole effect", Journal of Sound and Vibration In Press, (2010). 
2 D. J. O’Boy, E. P. Bowyer, and V. V. Krylov, "Damping of flexural vibrations in thin plates using one and two dimensional acoustic black hole effect", in 10th International Conference on Recent Advances in Structural Dynamics, RASD2010, Southampton, UK, 10-14th July (2010).

3 E. E. Ungar, "Loss factors of viscoelastically damped beam structures", Journal of the Acoustical Society of America 34, 1082-1089 (1962).

4 D. Ross, E. M. Kerwin, and E. E. Ungar, "Damping of plate flexural vibrations by means of viscoelastic laminae", in Structural Damping (Ruzicka, J. E., ed) 3, 44-87 (1959).

5 E. E. Ungar and E. M. J. Kerwin, "Plate damping due to thickness deformations in attached viscoelastic layers", Journal of the Acoustical Society of America 36, 386-392 (1964).

6 V. V. Krylov, "New type of vibration dampers utilising the effect of acoustic 'black holes"', Acta Acustica united with Acustica 90, 830-837 (2004).

7 V. V. Krylov and F. J. B. S. Tilman, “Acoustic 'black holes' for flexural waves as effective vibration dampers", Journal of Sound and Vibration 274, 605-619 (2004).

8 V. V. Krylov and R. E. T. B. Winward, "Experimental investigation of the acoustic black hole effect for flexural waves in tapered plates", Journal of Sound and Vibration 300, $43-49$ (2007).

9 F. Gautier, J. Cuenca, V. V. Krylov, and L. Simon, "Experimental investigation of the acoustic black hole effect for vibration damping in elliptical plates (abstract)", in Second Acoustical Society of America and European Acoustics Association joint conference (Congrs Acoustics'08), Paris, 24-29 June (2008).

10 M. A. Mironov, "Propagation of a flexural wave in a plate whose thickness decreases smoothly to zero in a finite interval", Soviet Physics - Acoustics 34, 318-319 (1988).

11 J. H. Gaines and E. Volterra, "Transverse vibrations of cantilever bars of variable cross section", Journal of the Acoustical Society of America 39, 674-679 (1966).

12 H. H. Mabie and C. B. Rogers, "Transverse vibrations of tapered cantilever beams with end support", Journal of the Acoustical Society of America 44, 1739-1741 (1968). 
13 H. H. Mabie and C. B. Rogers, "Transverse vibrations of double-tapered cantilever beams with end support and with end mass", Journal of the Acoustical Society of America 55, 986-991 (1974).

14 H. D. Conway, "Some special solutions for the flexural vibrations of discs of varying thickness", Ing.-Arch. 26, 408-410 (1958).

15 V. Pagneux and A. Maurel, "Lamb wave propagation in inhomogeneous elastic waveguides", Proceedings of the Royal Society London 458, 1913-1930 (2002).

16 V. Pagneux and A. Maurel, "Lamb wave propagation in elastic waveguides with variable thickness", Proceedings of the Royal Society 462, 1315-1339 (2006).

17 P. Marical, M. E. C. El-Kettani, and M. V. Predoi, "Guided waves in elastic plates with Gaussian section variation: Experimental and numerical results", Ultrasonics 47, 1-9 (2007).

18 M. E. C. El-Kettani, F. Luppe, and A. Guillet, "Guided waves in a plate with linearly varying thickness: experimental and numerical results", Ultrasonics 42, 807-812 (2004).

19 C. Prada, D. Clorennec, and D. Royer, "Power law decay of zero group velocity lamb modes", Wave Motion 45, 723-728 (2008).

20 A. H. Meitzler, "Backward-wave transmission of stress pulses in elastic cylinders and plates", Journal of the Acoustical Society of America 38, 835-842 (1965).

21 S. P. Timoshenko, Theory of Plates and Shells (New York: McGraw-Hill) (1959), p52, p290.

22 K. F. Graff, Wave Motion in Elastic Solids (Clarendon Press, Oxford) (1975), p251-258.

23 G. Z. Harris, "The normal modes of a circular plate of variable thickness", Quart. Journal of Mech. and Applied Math. 21, 331-327 (1967).

24 D. J. O'Boy and A. P. Dowling, "Tyre / road interaction noise: A 3d viscoelastic multilayer model of a tyre belt.", Journal of Sound and Vibration 322, 829-850 (2009).

25 M. Heckl, L. Cremer, and E. E. Ungar, Structure Borne Sound, 2nd Edition (SpringerVerlag) (1988), p153, 161-220.

26 C. W. de Silva, Vibration: Fundamentals and Practice (CRC Press) (1999), p384, 398- 
409.

27 J. Edward M. Kerwin, "Damping of flexural waves by a constrained viscoelastic layer", The Journal of the Acoustical Society of America 31, 952-962 (1959).

28 D. J. Mead, Passive Vibration Control (Wiley \& Sons, Chichester) (1998), p257, 359-387.

29 R. A. DiTaranto, "Theory of vibratory bending for elastic and viscoelastic layered finitelength beams", Journal of Applied Mechanics 32, 881-886 (1965).

30 J. Wang, "Generalized power series solutions of the vibration of classical circular plates with variable thickness", Journal of Sound and Vibration 202, 593-599 (1997).

31 N. K. Mandal, R. A. Rahman, and M. S. Leong, "Experimental study on loss factor for corrugated plates by bandwidth method", Ocean Engineering 31, 1313-1323 (2004). 


\section{List of Figures}

FIG. 1 Circular composite disc incorporating a quadratic power-law profile section in the center (not to scale), (a) illustration (b) notation for numerical model. 7

FIG. 2 Vibration amplitude $w(\omega, n=0, r) / p$ of a composite plate comprising an annulus with an undamped profiled indentation. The frequency dispersion curve is shown in (a) for different radial positions on the composite plate when excited in the $n=0$ mode. In (b) the displacement amplitude is shown for a single frequency of $1850 \mathrm{~Hz}$ and $n=0$, showing the nodal pattern created by the flexural waves traveling into the center of the plate, through the power-law profile and reflecting out from the free edge at the truncation point (the lighter the color, the higher the amplitude). . . . . . . . . . . . 14

FIG. 3 Driving point mobility measurements $(v / p)$ dB showing the cylindrical plate of constant thickness for numerical predictions against experimental measurements (color online) . . . . . . . . . . . . . . . . . . . . 16

FIG. 4 Driving point mobility measurements $(v / p)$ dB showing the annular plate of constant thickness with a central damped wedge indentation of power-law profile, numerical predictions and experimental measurements (color online).

FIG. 5 Estimates of the total loss factor $\eta_{\text {Total }}$ for three different circular plates by the half-power bandwidth method (a) Experimental measurements, (b) Numerical predictions (color online) . . . . . . . . . . . . . . . . . 18

FIG. 6 Predictions of the average peak point mobility measurements $\bar{v}=$ $\frac{1}{10} \sum_{k=1}^{k=10} v\left(\omega, \theta=0, r_{k}\right) / p\left(r_{k}\right) \mathrm{dB}$ (color online). . . . . . . 


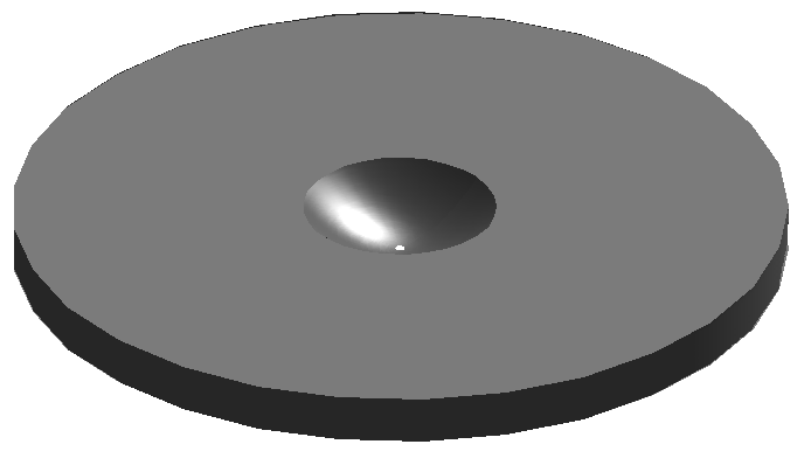




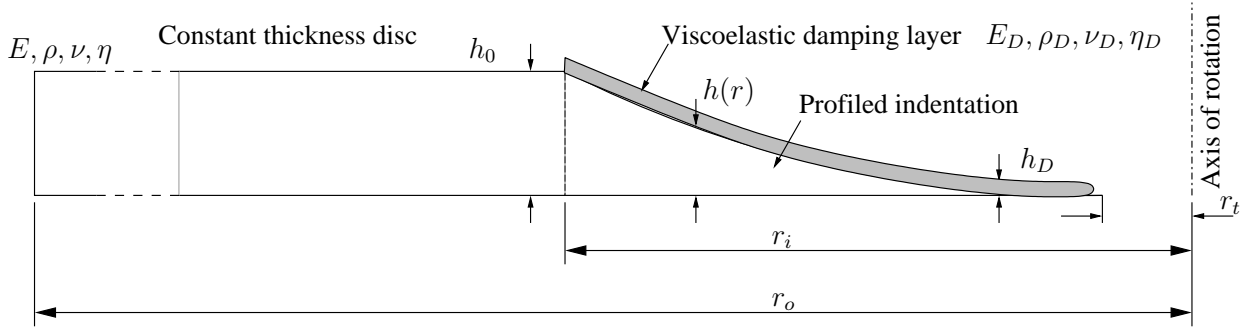




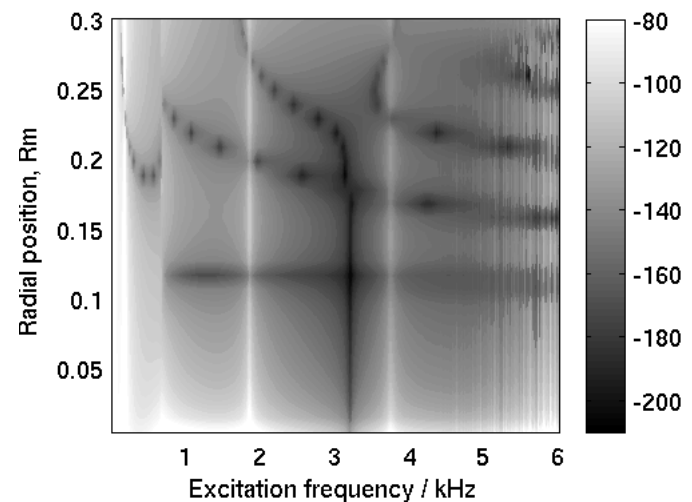




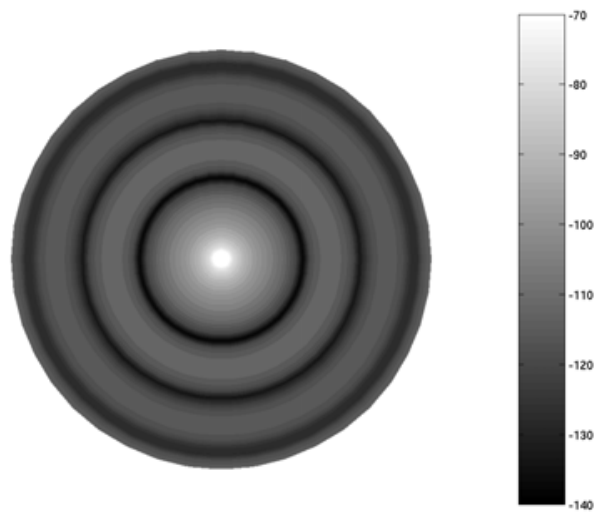




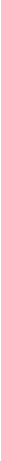




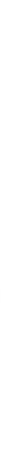




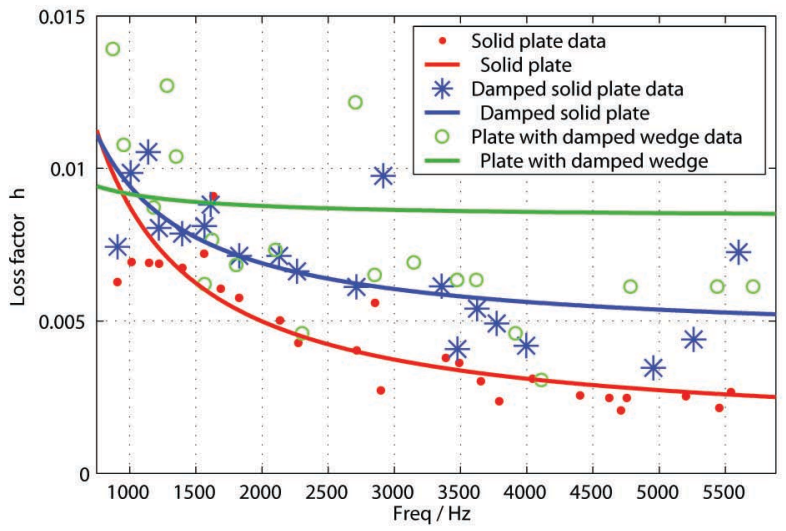




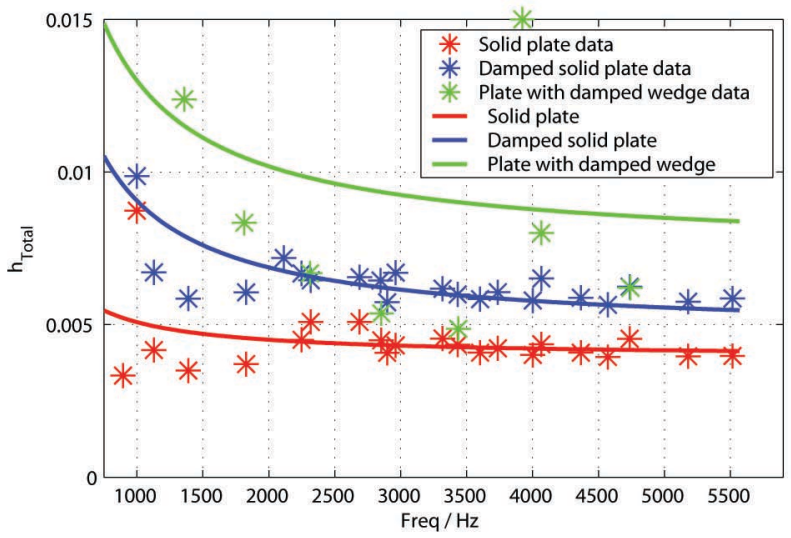


